\title{
GEOMETRÍA FRACTAL Y EUCLIDIANA APLICADA AL DIAGNÓSTICO DE GRADOS DE LESIÓN DE CÉLULAS DE CUELLO UTERINO
}

\section{EUCLIDIAN AND FRACTAL GEOMETRY APPLIED TO THE DIAGNOSIS OF LESIONS FROM CERVICAL CELLS}

\author{
Javier Rodríguez ${ }^{1}$, Miguel Sánchez ${ }^{2}$, Freddy Barrios ${ }^{3}$, Yolanda Soracipa ${ }^{4}$
}

\begin{abstract}
${ }^{1}$ Med Director del Grupo Insight. Centro de Investigaciones Clínica del Country, Carrera 79B No. 51-16 sur Int 5, Apt 102, Bogotá, Colombia, e-mail: grupoinsight2025@yahoo.es; ${ }^{2}$ Med Director de Investigación y Proyección Social, Líder grupo de investigación Salud Sexual y Reproductiva. Universidad El Bosque, Carrera 7b Bis Nº 132-11, Bogotá, Colombia, e-mail: sanchezcmiguel@unbosque.edu.co; ${ }^{3}$ Estudiante de la Maestría de Salud Sexual y Reproductiva. Universidad El Bosque, Carrera 7b Bis No 132-11, Bogotá, Colombia, e-mail: fbarriosa@unbosque.edu.co; ${ }^{4}$ Lcda. Investigadora Grupo Insight. Centro de Investigaciones Clínica del Country, Bogotá, Colombia, e-mail: grupoinsight2025@yahoo.es
\end{abstract}

Rev. U.D.C.A Act. \& Div. Cient. 20(1): 13-21, Enero Junio, 2017

\section{RESUMEN}

Previamente, se desarrolló una metodología diagnóstica para lesiones preneoplásicas y neoplásicas de células de cuello uterino, a partir de medidas euclidianas y fractales simultáneas. En este trabajo, el objetivo era confirmar la concordancia diagnóstica de la metodología en células normales y en diferentes estadios de lesión celular. Se tomaron fotografías de 60 células del epitelio escamoso cervical: 10 normales, 10 ASCUS, 20 con lesión intraepitelial de bajo grado (LEIBG) y 20 con lesión de alto grado (LEIAG). Se realizaron medidas de dimensión fractal y del espacio de ocupación de la superficie y el borde del núcleo y citoplasma en el espacio fractal de Box Counting, estableciendo su diagnóstico físico-matemático. Las medidas de la superficie del núcleo estuvieron para normalidad, entre 305 y 651; para ASCUS, entre 1293 y 4588; para LEIBG, entre 986 y 4873 y para LEIAG, entre 567 y 2311. La resta de las fronteras Citoplasma-Núcleo, se encontró entre 238 y 477, para normalidad; entre 185 y 417, para ASCUS; entre 131 y 342, para LEIBG y entre 43 y 117, para LEIAG. Fueron hallados valores de sensibilidad y especificidad del $100 \%$; la razón de probabilidad fue de 0 y el coeficiente kappa de 1 . Se confirmó la concordancia diagnóstica a nivel clínico del método físico-matemático, cuantificando de manera objetiva y reproducible el grado de lesión de células de cérvix y estableciendo un diagnóstico objetivo para las células ASCUS, a partir de medidas fractales y euclidianas simultáneas, que mejora los métodos cualitativos de clasificación.

Palabras clave: ASCUS, LEIBG, LEIAG, cáncer de cérvix, citología.

\section{SUMMARY}

A diagnostic methodology for preneoplastic and neoplastic lesions of cervical cells from simultaneous fractals and euclidean measures was developed. In this work the objective was to confirm the diagnostic concordance of the methodology in normal cells and in different stages of progression to disease. For this, 60 photographs of cervical squamous epithelium cells were taken: 10 normal, 10 ASCUS, 20 with low grade - squamous intraepithelial lesion (L-SIL) and 20 high-grade lesion (H-SIL). Measures of fractal dimension and space occupancy of the surface and the edge of the nucleus and cytoplasm were performed in Box Counting Fractal space, establishing their physical-mathematical diagnosis. Measurements of the nucleus surface for normality were between 305 and 651, for ASCUS between 1293 and 4588, for L-SIL, between 986 and 4873 and for H-SIL between 567 and 2311. The subtraction of Cytoplasm-Nucleus border was found between 238 and 477 for normality; between 185 and 417 for ASCUS; between 131 and 342 for L-SIL and between 43 and 117 for H-SIL, finding sensitivity and specificity of $100 \%$, likelihood ratio of 0 and kappa coefficient of 1 . Conclusions: the diagnostic concordance at clinical level of the physical-mathematical method was confirmed, objective and reproducible quantifying the degree of injury of cervical cells and establishing an objective diagnosis for ASCUS cells from simultaneous fractals and euclidean measures, which improves qualitative classification methods.

Key words: ASCUS, L-SIL, H-SIL, cervix cancer, cytology. 


\section{INTRODUCCIÓN}

La geometría euclidiana estudia los objetos regulares mediante medidas, como la longitud, el área o el volumen. Aplicar estas medidas a objetos irregulares lleva a resultados paradójicos, como lo mostró Mandelbrot (2000), al tratar de utilizar la geometría euclidiana, en la medición de la longitud de la costa de Gran Bretaña; por lo cual, propuso la geometría fractal, como una nueva forma de medir este tipo de objetos irregulares.

Los objetos naturales, que incluyen las estructuras del cuerpo humano, tienen, generalmente, estructura irregular (Goldberger et al. 1990). A los objetos irregulares, se les ha denominado fractales, los cuales, se han clasificado en tres tipos: abstracto, estadístico y salvaje; esta última clase es a la que, usualmente, corresponden los objetos naturales y se mide con la dimensión fractal de Box Counting. La dimensión fractal es una medida de la geometría fractal, que muestra el grado de irregularidad del objeto medido (Peitgen et al. 1992).

Las pacientes con cáncer cervical son, por lo general, diagnosticadas en etapas tempranas, lo que hace que sus tratamientos sean exitosos, tengan muy buena prognosis y que puedan ser tratadas únicamente con cirugía; sin embargo, un diagnóstico tardío aumenta de forma dramática las probabilidades de metástasis y mortalidad. Más del $20 \%$ de pacientes presentan enfermedad extrauterina en el diagnóstico, de acuerdo con Hoekstra et al. (2009) y, aproximadamente, del 7 al $12 \%$ de las pacientes presentan metástasis pélvica o en los nodos linfáticos, para-aórticos (McMeekin et al. 2001; Adab et al. 2004; Chan \& Kapp, 2007). Estas cifras revelan la importante y urgente necesidad de generar metodologías que ayuden en el diagnóstico oportuno y confiable de la enfermedad, lo que puede llevar a una reducción significativa de riesgo para las pacientes, según Gakidou et al. (2008).

La citología cérvico vaginal (CCV) sigue siendo el examen más utilizado para la prevención del cáncer cérvico-uterino (NIH, 1996). Mediante el seguimiento de la evaluación de la CCV es posible detectar la presencia de células con lesiones, que indiquen el desarrollo de la enfermedad; sin embargo, su evaluación se fundamenta en observaciones cualitativas, lo que ha imposibilitado un consenso, a nivel mundial, para su diagnóstico y se ha mostrado que este examen presenta una sensibilidad promedio de solo 51\% (Nanda et al. 2000). En la evaluación clínica convencional, se utilizan medidas, que incluyen, la observación del tamaño del núcleo y del citoplasma (Schmidt et al. 2008), así como la observación simultánea de varios parámetros cualitativos; no obstante, se presentan problemas de reproducibilidad inter e intra observador (Nanda et al. 2000; Lacruz, 2003; Schmidt et al. 2008; Geisinger et al. 2007).

En busca de una mejor reproducibilidad y objetividad en la evaluación de este examen, se han desarrollado estudios que aplican metodologías fractales para su estudio (Rodríguez et al. 2006; Rodríguez et al. 2010). Medidas similares se han aplicado a otro tipo de estructuras neoplásicas o preneoplásicas, tal es el caso de la aplicación de la geometría fractal al análisis de mamografías (Lefebvre \& Benali, 1995; Pohlman et al. 1996; Sankar \& Thomas, 2010; Stępień \& Stępień, 2010), de cáncer invasivo de vejiga (Luzi et al. 1999), de la mucosa oral (Landini \& Rippin, 1993) y de tejido ocular (Gazit et al. 1995; De Arruda et al. 2013; Klatt et al. 2013; Metze, 2013).

Recientemente, han sido desarrollados nuevos estudios morfométricos de células de cuello uterino con geometría fractal (Rodríguez, 2011; Rodríguez et al. 2013; Rodríguez et al. 2014a; Prieto et al. 2014). Consisten en evaluaciones matemáticas de carácter objetivo y reproducible del grado de irregularidad de las células del epitelio escamoso de cuello uterino, identificadas microscópicamente como normales (Figura 1 a), atipias escamosas de significado indeterminado (ASCUS) (Figura 1 b), lesiones escamosas intraepiteliales de bajo grado (LEIBG) (Figura $1 \mathrm{c}$ ) y lesiones escamosas intraepiteliales de alto grado (LEIAG) (Figura $1 \mathrm{~d}$ ), basados en medidas fractales del núcleo y citoplasma celular (Tabla 1). Esta perspectiva físico-matemática ha permitido establecer simulaciones de rutas de evolución celular, desde normalidad hasta ASCUS (Rodríguez et al. 2013) y LEIBG (Rodríguez et al. 2014a), que se pueden observar en la práctica clínica. Prieto et al. (2014) desarrollaron un método diagnóstico de la citología de cuello uterino, basado en geometría fractal y euclidiana, que diferencia matemáticamente células normales, LEIBG y LEIAG, diferenciando ASCUS, que pueden tener las relaciones matemáticas de normalidad o de enfermedad. Esto fue posible, gracias al establecimiento de medidas de borde y de superficie de núcleo y citoplasma, en el contexto del espacio fractal, generalizado de Box Counting.

El propósito de este trabajo es confirmar la capacidad diagnóstica y aplicabilidad clínica de la metodología fundamentada en la geometría fractal y euclidiana desarrollada previamente por Prieto et al. (2014), para evaluar células de cuello uterino normales ASCUS, LEIBG y LEIAG. 
Tabla 1. Definiciones desde las que se fundamenta la metodología del presente trabajo.

\begin{tabular}{|l|l|}
\hline \multicolumn{1}{|c|}{ Concepto } & \multicolumn{1}{c|}{ Definición } \\
\hline $\begin{array}{l}\text { Dimensión fractal: Me dida numérica adimen- } \\
\text { sional del grado de irregularidad de un objeto. } \\
\begin{array}{l}\text { Para esta investigación, se utilizará la definición de } \\
\text { dimensión fractal de Box-Counting, empleada para } \\
\text { fractales salvajes. }\end{array}\end{array}$ & $\begin{array}{l}\text { Ecuación 1: Dimensión fractal de Box-Counting } \\
\text { Siendo: N, el número de cuadros que contiene el contorno del obje- } \\
\text { to; K, el grado de partición de la cuadrícula y, D, la dimensión fractal. }\end{array}$ \\
\hline Borde del objeto & $\begin{array}{l}\text { Número de espacios ocupados por el borde de los objetos (núcleo y } \\
\text { citoplasma), en la rejilla de 2x2 pixeles (Figura 2b). }\end{array}$ \\
\hline Superficie del objeto & $\begin{array}{l}\text { Se denomina como el número de pixeles que ocupan los objetos, } \\
\text { núcleo y citoplasma (Figura 2a). }\end{array}$ \\
\hline Resta fronteras Citoplasma-Núcleo & $\begin{array}{l}\text { Diferencia entre los valores hallados en la frontera del citoplasma y el } \\
\text { núcleo en la rejilla. }\end{array}$ \\
\hline
\end{tabular}

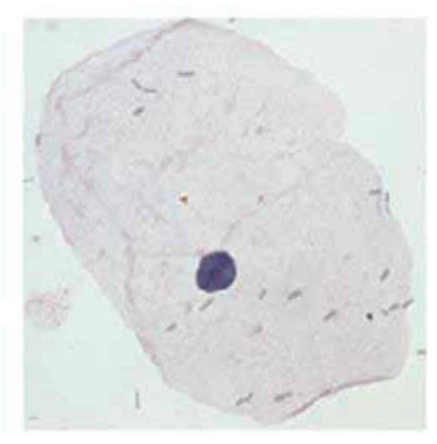

(a)

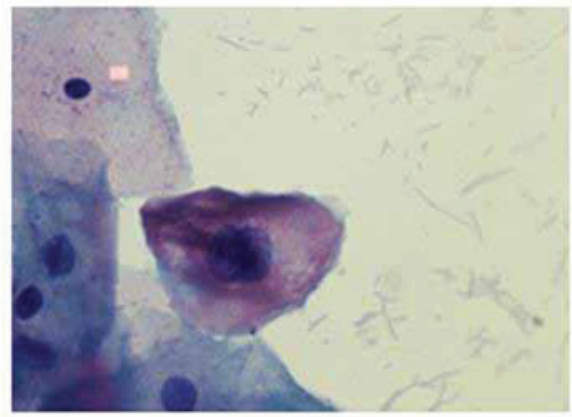

(c)

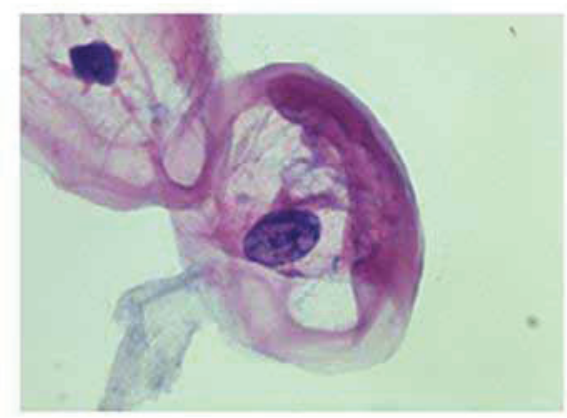

(b)

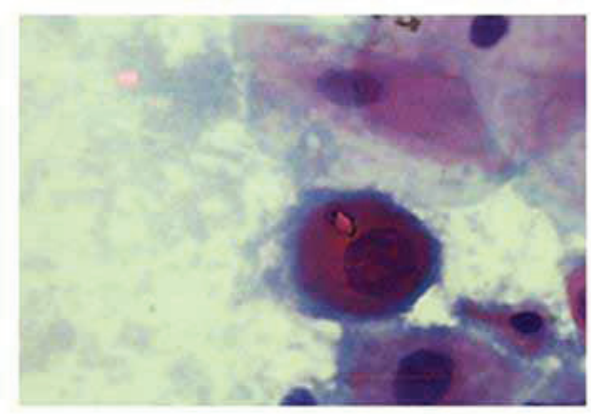

(d)

Figura 1. Ejemplos de las células medidas, en su orden: a) célula normal, b) célula Ascus, c) célula LEI bajo grado (LEIBG) y d) célula LEI Alto grado (LEIAG).

\section{MATERIALES Y MÉTODOS}

Citologías de cuello uterino de 60 mujeres, con edades entre 21 y 58 años, fueron seleccionadas, provenientes de archivos de datos del grupo Insight, presentando reportes de citología normal y con diferentes grados de lesión hasta carcinoma, de acuerdo con los parámetros convencionales evaluados por un patólogo experto. Los diagnósticos clínicos de las muestras fueron enmascarados, para el desarrollo de un estudio ciego. 
Se seleccionó una célula representativa de cada muestra; para esto, se observaron, mediante microscopio, los extendidos de las citologías, en busca de células, cuyas características concordaran con la evaluación clínica de cada una de las clasificaciones analizadas: normal, ASCUS, LEIBG y LEIAG, de acuerdo a la experticia del patólogo. Dichas células fueron fotografiadas con cámara digital (Nikon), a través de microscopio (Nikon Eclipse ع200), con aumento de 100X y analizadas con un editor de imágenes, superponiendo rejillas con cuadros de 2 y 4 pixeles de lado, para el cálculo de la dimensión fractal de los objetos definidos, que son núcleo y citoplasma sin núcleo de cada célula, empleando un software previamente desarrollado por el Grupo Insight, que realiza una medición de la superficie (Figura 2a) y el borde de núcleo (Figura 2 b) y citoplasma con las rejillas que sean definidas y que, asimismo, realiza el cálculo de la dimensión fractal. Se cuantificó el número de cuadros que ocupa la frontera de cada uno de los objetos medidos; seguidamente, se calculó el número de pixeles que ocupa la superficie de los objetos definidos (Tabla 1). A continuación, se calculó la resta entre los valores del borde del núcleo y citoplasma.

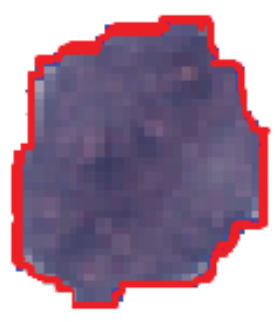

(a)

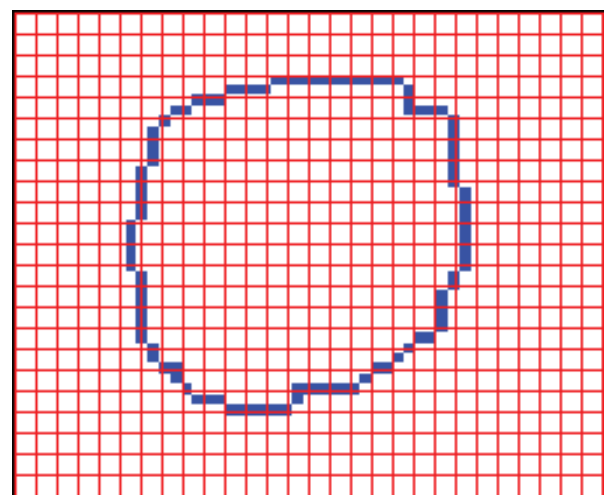

(b)

Figura 2. Medidas de las células. a) Superficie del objeto, número de pixeles del área del objeto, en este ejemplo, el número de pixeles dentro del contorno de la figura (línea roja) del núcleo de una célula y b) Magnificación de la imagen de la superposición de la rejilla de $2 \times 2$ pixeles, para medir el número de cuadros que ocupa el borde (línea azul) del núcleo de una célula.

Posteriormente, se estableció el diagnóstico físico-matemático, a partir de los siguientes criterios, establecidos en el trabajo previo de Prieto et al. (2014): las células normales, se caracterizan por presentar superficies nucleares menores o iguales a 735 y valores mayores o iguales a 161 en la resta de las fronteras Citoplasma-Núcleo; las células LEIBG presentan valores mayores o iguales a 972, en la superficie del núcleo y un valor mayor a 130, en la resta de las fronteras Citoplasma-Núcleo y las células LEIAG, se caracterizan por tener valores menores a 120, en la resta de las fronteras Citoplasma-Núcleo. La diferenciación entre LEIBG y LEIAG, se realiza solo con la resta de las fronteras Citoplasma-Núcleo. El rango 120-130 en el valor de la resta de las fronteras Citoplasma-Núcleo corresponde a la evolución entre células LEIBG Y LEIAG.

Aspectos éticos: Este trabajo, se hizo de acuerdo con las guías éticas del artículo 11 de la resolución 008430 de 1993, del Ministerio de Salud de Colombia, donde está clasificado en la categoría de investigación sin riesgo, pues se realizan cálculos matemáticos sobre resultados de exámenes de la práctica clínica, que han sido prescritos médicamente, protegiendo la integridad y anonimato de los participantes y que provienen de bases de datos de investigaciones previas.
Análisis estadístico: Se desenmascararon los diagnósticos citopatológicos de las muestras, las cuales, fueron tomadas como Gold-estándar, para desarrollar el análisis estadístico. Para efectos del análisis estadístico, se estableció que las células diagnosticadas histológicamente como LEIBG o LEIAG forman un solo grupo de células patológicas. Para el cálculo de los verdaderos positivos, se tomaron las células que fueron evaluadas como patológicas, tanto por la metodología matemática como por el diagnóstico histológico; los verdaderos negativos correspondieron a las células que fueron evaluadas como normales, tanto por la metodología matemática como por el diagnóstico histológico; para los falsos positivos, se tomaron las células diagnosticadas histológicamente como normales, pero matemáticamente como patológicas y los falsos negativos correspondieron a las células evaluadas matemáticamente como normales, pero con diagnóstico histológico de lesión. Una vez hallados estos valores fue calculada la sensibilidad y la especificidad, mediante una tabla de contingencia de $2 \times 2$, entendiendo la primera, como la proporción de enfermos adecuadamente identificados y, la segunda, como la proporción de pacientes sanos adecuadamente identificados. 
Las células clasificadas como ASCUS, al no tener un diagnóstico histológico específico de lesión o normalidad, fueron excluidas del análisis estadístico. Posteriormente, se calculó la razón de probabilidad y el coeficiente kappa.

\section{RESULTADOS Y DISCUSIÓN}

Este es el primer trabajo de aplicación clínica del diagnóstico fractal y euclidiano de células de cuello uterino observadas en citologías, desde normalidad hasta LEIAG. Se confirmó la capacidad de la metodología para diferenciar normalidad, LEIBG y LEIAG, cuantificando la evolución del proceso preneoplásico, a partir de medidas de ocupación celular, en el espacio generalizado del Box-Counting. Las medidas morfométricas desarrolladas revelaron una auto-organización del fenómeno, que permite establecer una evaluación objetiva y reproducible de cada célula, constituyéndose en una herramienta útil de aplicación clínica. Estas medidas matemáticas hechas a la estructura celular en el desarrollo preneoplásico y neoplásico, permiten superar las dificultades de reproducibilidad de los sistemas de clasificación actual, como el Sistema Bethesda.
Se encontró que la superficie del núcleo de las células normales del epitelio escamoso cervical varió entre 305 y 651, para normalidad (Tabla 2); entre 1293 y 4588, para ASCUS; entre 986 y 4873, para LEIBG y entre 567 y 2311, para LEIAG. Estos resultados permiten diferenciar normalidad de ASCUS y de LEIBG; sin embargo, no diferencian normalidad de LEIAG.

Las medidas de la frontera del núcleo de células normales presentaron valores entre 37 y 54; las ASCUS, entre 78 y 131; las LEIBG, entre 72 y 158 y las LEIAG, entre 68 y 105.

Las medidas de la frontera del citoplasma, se encontraron entre 275 y 523, para normalidad; entre 263 y 519, para las células ASCUS; entre 248 y 465, para LEIBG y entre 111 y 203, para LEIAG.

La resta entre los valores de las medidas de la frontera del citoplasma y del núcleo presentó valores entre 238 y 477 , para normalidad; entre 185 y 417, para ASCUS; entre 131 y 342, para LEIBG y entre 43 y 117, para LEIAG. Esta medida permite diferenciar, matemáticamente, las células normales de las LEIAG.

Tabla 2. Medidas físico-matemáticas de 20 células: 5 normales, 5 ASCUS, 5 LEIBG y 5 LEIAG, junto con el diagnóstico convencional establecido por patólogo experto y el diagnóstico físico-matemático. Se eligieron las muestras más representativas de cada uno de los estados.

\begin{tabular}{|c|c|c|c|c|c|c|}
\hline No. & $\begin{array}{c}\text { Diagnóstico } \\
\text { convencional }\end{array}$ & Superficie núcleo & Frontera núcleo & $\begin{array}{c}\text { Frontera } \\
\text { citoplasma }\end{array}$ & $\begin{array}{c}\text { Resta fronteras } \\
\text { C-N }\end{array}$ & $\begin{array}{c}\text { Diagnóstico } \\
\text { matemático }\end{array}$ \\
\hline 1 & normal & 407 & 37 & 275 & 238 & Normal \\
\hline 2 & normal & 305 & 46 & 523 & 477 & Normal \\
\hline 3 & normal & 651 & 53 & 307 & 254 & Normal \\
\hline 4 & normal & 342 & 39 & 464 & 425 & Normal \\
\hline 5 & normal & 466 & 54 & 511 & 457 & Normal \\
\hline 6 & ASCUS & 1293 & 78 & 263 & 185 & LEIBG \\
\hline 7 & ASCUS & 2358 & 102 & 519 & 417 & LEIBG \\
\hline 8 & ASCUS & 3101 & 131 & 437 & 306 & LEIBG \\
\hline 9 & ASCUS & 2506 & 95 & 285 & 190 & LEIBG \\
\hline 10 & ASCUS & 4588 & 121 & 489 & 368 & LEIBG \\
11 & LEIBG & 986 & 117 & 248 & 131 & LEIBG \\
\hline 12 & LEIBG & 4302 & 158 & 465 & 307 & LEIBG \\
\hline 13 & LEIBG & 3693 & 120 & 328 & 208 & LEIBG \\
\hline 14 & LEIBG & 2565 & 72 & 279 & 207 & LEIBG \\
\hline 15 & LEIBG & 4873 & 99 & 441 & 342 & LEIBG \\
\hline 16 & LEIAG & 743 & 68 & 111 & 43 & LEIAG \\
\hline 17 & LEIAG & 2311 & 105 & 203 & 98 & LEIAG \\
\hline 18 & LEIAG & 1735 & 85 & 175 & 90 & LEIAG \\
\hline 19 & LEIAG & 567 & 93 & 139 & 46 & LEIAG \\
\hline 20 & LEIAG & 1743 & 77 & 194 & 117 & LEIAG \\
\hline
\end{tabular}


Con base en estos valores, se determinó el diagnóstico físico-matemático de cada célula, encontrando que las células diagnosticadas como normales con el diagnóstico matemático fueron consideradas normales, de acuerdo con los parámetros convencionales y lo mismo ocurrió con las LEIBG y LEIAG, presentando el mismo diagnóstico, tanto por el patólogo como por el método matemático. Los valores de las células ASCUS de este estudio, se comportaron matemáticamente como LEIBG, resultado que concuerda con los valores hallados por Prieto et al. (2014).

Convencionalmente, para la determinación del estado celular, uno de los criterios usados en las metodologías de clasificación es la observación cualitativa del tamaño del núcleo y del citoplasma (Schmidt et al. 2008; Landy et al. 2015); sin embargo, este tipo de observación implica problemas de reproducibilidad (Nanda et al. 2000; Lacruz, 2003; Schmidt et al. 2008; Geisinger et al. 2007).

Además, la clasificación ASCUS, implementada por el sistema Bethesda, constituye un espacio de clasificación de células que, por sus características, evaluadas cualitativamente, no se pueden definir claramente como normales, LEIBG o LEIAG.

En contraposición, con esta metodología es posible establecer, cuantitativamente, el orden geométrico de la irregularidad de la frontera y las diferencias de magnitud de superficie nuclear, con las cuales, se pueden determinar diferencias cuantitativas de toda la evolución, entre normalidad hasta distintos grados de lesión, incluyendo las células ASCUS, cuantificando su posible relación con la evolución a estados de mayor gravedad. Al fundamentarse en una armonía geométrica subyacente de la estructura celular, constituye una herramienta diagnóstica, que puede unificar los sistemas de clasificación cualitativa de la citología.

Un estudio realizado por Nanda et al. (2000) encontró que la sensibilidad de la CCV era del $51 \%$, mientras que su especificidad era del $98 \%$. Más aun, el valor predictivo positivo (VPP) de discariosis severa/invasiva y citología de neoplasia glandular fue 34 y $12 \%$, respectivamente; el VPP de discariosis grave, entendida como una LEIAG o displasia severa, fue del $4 \%$ (Landy et al. 2015). La presente investigación halló valores de sensibilidad y de especificidad del $100 \%$, una razón de probabilidad de cero y un coeficiente Kappa de 1, mostrando la mayor concordancia diagnóstica con el Gold Standard. Considerando los hallazgos mencionados y dada la objetividad de la metodología presentada, ligada a la evaluación de un orden geométrico intrínseco a la estructura celular, se considera que podría ser aplicable a cada caso particular, al margen de análisis poblacionales o factores de riesgo.
Actualmente, la utilización de la geometría fractal en medidas de la superficie celular ha cobrado atención, para caracterizar la progresión de estas células hacia cáncer (Guz et al. 2015; Bartrés et al. 2016), así como para evaluar tejidos (Nicolis et al. 2017); sin embargo, aún existen dificultades técnicas (Sokolov et al. 2017) y de aplicabilidad, pues varios de estos métodos se basan en la toma de imágenes, a partir de Atomic force microscopy (AFM) o de tecnología que no está disponible en muchos países en vía de desarrollo, por lo que metodologías, como la utilizada en el presente trabajo, permiten hacer una evaluación más directa y de más fácil aplicación, para que pueda ser utilizada de forma masiva, sin tener que depender del avance tecnológico.

Esta nueva metodología diagnóstica, desarrollada por Rodríguez et al., se fundamenta en metodologías previas, basadas en la aplicación de la geometría fractal (Rodríguez et al. 2006; Rodríguez et al. 2010; Rodríguez, 2011; Rodríguez et al. 2014a), evidenciando que la dimensión fractal, evaluada aisladamente, no es suficiente para establecer diferencias matemáticas entre normalidad y LEIBG (Rodríguez et al. 2006), por lo que se hizo necesario crear el concepto de Armonía Matemática Intrínseca Celular y Variabilidad de la Dimensión Fractal, para diferenciar ambos estados (Rodríguez et al. 2010; Rodríguez, 2011).

Posteriormente, se comenzó a emplear, de manera simultánea, geometría fractal y euclidiana en eritrocitos, por Correa et al. (2012), con el fin de implementar medidas objetivas del borde y la superficie, a partir del espacio ocupado por dichas estructuras, en el espacio generalizado de Box Counting. Este nuevo procedimiento aplicado a células de cérvix evidenció que el aumento del valor del borde nuclear indica que se está ante un caso de mayor gravedad, hecho que es independientemente de si la célula es evaluada de forma cualitativa, como ASCUS o LEIBG (Rodríguez et al. 2014b).

Gracias a estos hallazgos fue posible establecer la metodología diagnóstica, cuya aplicabilidad se comprueba en el presente trabajo de Prieto et al. (2014) y que sirvió también como fundamento para una generalización, en la cual, se establecen todas las posibles trayectorias de evolución de las células de cuello uterino, desde normalidad hasta neoplasia, de aplicabilidad diagnóstica y preventiva (Velásquez et al. 2015).

El presente estudio no consideró relaciones causa-consecuencia temporal; en contraposición, se fundamentó en el concepto de ventanas temporales, enunciado por Prigogine, para aludir a fenómenos que no pueden ser descritos mediante medidas continuas (Fernández, 1990). Desde esta perspectiva acausal, la aplicación de la metodología permite incluir y diagnosticar todas las posibles alteraciones celulares, independientemente de la edad, de los factores de ries- 
go o de cualquier análisis poblacional, siendo aplicables a cada caso particular.

El presente estudio confirmó la aplicabilidad clínica y la reproductibilidad del método físico-matemático, a partir de medidas fractales y euclidianas simultáneas, al cuantificar, de manera objetiva y reproducible, el grado de lesión de células de cérvix. La metodología permite, además, establecer un diagnóstico objetivo para las células ASCUS, permitiendo superar su indeterminación diagnóstica, lo cual, tendría implicaciones importantes en el diagnóstico oportuno y la reducción de la morbimortalidad.

Agradecimientos: Agradecemos a la Universidad El Bosque, especialmente, a su División de Investigaciones, por el apoyo brindado a la investigación. Un agradecimiento especial al Dr. Héctor Posso, Director Científico de la Liga contra el Cáncer, por su apoyo a las investigaciones; también a las doctoras Catalina Correa y Signed Prieto, investigadoras del Grupo Insight, por el manejo del software y ayuda en el análisis de datos. Asimismo, al Centro de Investigaciones de la Clínica del Country por el apoyo a las investigaciones. Financiación: La presente investigación fue financiada por la División de Investigaciones de la Universidad El Bosque; proyecto PIC2014-19. Conflicto de interés: El manuscrito fue preparado y revisado con la participación de todos los autores, quienes declaramos que no existe conflicto de intereses, que ponga en riesgo la validez de los resultados presentados.

\section{BIBLIOGRAFÍA}

1. ADAB, P.; MCGHEE, S.; YANOVA, J.; WONG, CH.; HEDLEY, A. 2004. Effectiveness and efficiency of opportunistic cervical cancer screening comparison with organized screening. Med Care. 42:600-609.

2. BARTRÉS, A.; OLIVER, S.; PELLICER, B.; OLIVER, L.; CAMPO, V.; BARRIOS, M.; ARANA, E.; GONZÁLEZ, V. 2016. Algorithm programming for 3D fractal dimension evaluation. Global Medical Engineering Physics Exchanges/Pan American Health Care Exchanges (GMEPE/PAHCE), Madrid, p.1-4.

3. CHAN, J.K.; KAPP, D.S. 2007. Role of complete lymphadenectomy in endometrioid uterine cancer. Lancet Oncol. 8:831-841.

4. CORREA, C.; RODRÍGUEZ, J.; PRIETO, S.; BERNAL, P.; OSPINO, B.; MUNÉVAR, A.; ÁLVAREZ, L.; MORA, J.; VITERY, S. 2012. Geometric diagnosis of erythrocyte morphophysiology. J. Med. Med. Sci. 3(11):715-720.

5. DE ARRUDA, P.F.F.; GATTI, M.; FACIO, F.N.; DE ARRUDA, J.G.F.; MOREIRA, R.D.; MURTA, L.O. Jr.; DE
ARRUDA, L.F.; DE GODOY, M.F. 2013. Quantification of fractal dimension and Shannon's entropy in histological diagnosis of prostate cancer. BMC Clin. Pathol. 13:6.

6. FERNÁNDEZ, A. 1990. Introducción. En: Fernández, A. (ed.). Orden y Caos. Barcelona: Prensa Científica S.A. p. $4-8$

7. GAKIDOU, E.; NORDHAGEN, S.; OBERMEYER, Z. 2008. Coverage of cervical cancer screening in 57 countries: Low average levels and large inequalities. PLoS Med. 5(6):132.

8. GAZIT, Y.; BERK, D.A.; LUNIG, M.; BAXTER, L.T.; JAIN, R.K. 1995. Scale - invariant behavior and vascular network formation in normal and tumor tissue. Phys. Rev. Lett. (75):2428-2431.

9. GEISINGER, K.R.; VRBIN, C.; GRZYBICKI, D.M.; WAGNER, P.; GARVIN, A.J.; RAAB, S.S. 2007. Interobserver variability in human papillomavirus test results in cervico vaginal cytologic specimens interpreted as atypical squamous cells. Am. J. Clin. Pathol. 128(6), 1010-1014.

10. GOLDBERGER, A.; RIGNEY, D.R.; WEST, B. 1990. Caos y fractales en la fisiología humana. Investigación y Ciencia. 163:32-38.

11. GUZ, N.V.; DOKUKIN, M.E.; WOODWORTH, C.D.; CARDIN, A.; SOKOLOV, I. 2015. Towards early detection of cervical cancer: Fractal dimension of AFM images of human cervical epithelial cells at different stages of progression to cancer. Nanomedicine. 11(7):16671675.

12. HOEKSTRA, A.V.; KIM, R.J.; SMALL, J.R.; RADEMAKER, A.W.; HELENOWSKI, I.B.; SINGH D.K.; SCHINK, J.C.; LURAIN J.R. 2009. FIGO stage IIIC endometrial carcinoma: prognostic factors and outcomes. Gynecol Oncol. 114:273-278.

13. KLATT, J.; GERICH, C.; GRÖBE, A.; OPITZ, J.; SCHREIBER, J.; HANKEN, H.; SALOMON, G.; HEILAND, M.; KLUWE, L.; BLESSMANN, M. 2013. Fractal dimension of time-resolved autofluorescence discriminates tumor from healthy tissues in the oral cavity. $\mathrm{J}$. Craniomaxillofac Surg. 42(6):852-854.

14. LACRUZ, C. 2003. Nomenclatura de las lesiones cervicales de Papanicolau a Bethesda 2001. Rev. Esp. Patol. 36(1):5-10. 
15. LANDINI, G.; RIPPIN, J.W. 1993. Fractal dimensions of epithelial-connective tissue interfaces in premalignant and malignant epithelial lesions of the floor of mouth. Anal. Quant. Cytol. Histol. 15(2):144-149.

16. LANDY, R.; CASTANON, A.; HAMILTON, W.; LIM, A.W.; DUDDING, N.; HOLLINGWORTH, A.; SASIENI, P.D. 2015. Evaluating cytology for the detection of invasive cervical cancer. Cytopathology. 27(3):201-209.

17. LEFEBVRE, F.; BENALI, H. 1995. A fractal approach to the segmentation of microcalcifications in digital mammograms. Med. Phys. 22:381-390.

18. LUZI, P.; BIANCIARDI, G.; MIRACCO, C.; DE SANTI, M.M.; DEL VECCHIO, M.T.; ALIA, L.; TOSI, P. 1999. Fractal analysis in human pathology. Ann. NY Acad. Sci. 879:255-257.

19. MANDELBROT, B. 2000. ¿Cuánto mide la costa de Bretaña?. En: Mandelbrot B. (ed.) Los Objetos Fractales. Barcelona: Tusquets Eds. S.A.; p.27,50.

20. MCMEEKIN, D.S.; LASHBROOK, D.; GOLD, M.; JOHNSON, G.; WALKER, J.L.; MANNEL, R. 2001. Analysis of FIGO Stage IIIc endometrial cancer patients. Gynecol Oncol. 81:273-278.

21. METZE, K. 2013. Fractal dimension of chromatin: potential molecular diagnostic applications for cancer prognosis. Expert Rev. Mol. Diagn. 13(7):719-735.

22. NANDA, K.; MCCRORY, D.C.; MYERS, E.R.; BASTIAN, L.A.; HASSELBLAD, V.; HICKEY, J.D.; MATCHAR, D. 2000. Accuracy of the Papanicolaou test in screening for and follow-up of cervical cytologic abnormalities: a systematic review. Ann. Intern. Med. 132:810-819.

23. NHI. 1996. Consens Statement. 14(1): 1-38

24. NICOLIS, O.; KISEĹÁK, J.; PORRO, F.; STEHLÍK, M. 2017. Multi-fractal cancer risk assessment. Stoch. Anal. Appl. 35(2):237-256.

25. PEITGEN, H.; JURGENS, H.; SAUIPE, D. 1992. Chaos and fractals; new frontiers of science. New York: Springer. p.192-194.

26. POHLMAN, S.; POWELL, K.; OBUCHOWSKI, N.A.; CHILCOTE, W.A.; GRUNDFEST, S. 1996. Quantitative classification of breast tumors in digitized mammograms. Med Phys. 23:1337-1345.
27. PRIETO, S.; RODRÍGUEZ, J.; CORREA, C.; SORACIPA, Y. 2014. Diagnosis of cervical cells based on fractal and Euclidian geometrical measurements: Intrinsic Geometric Cellular Organization. BMC Medical Physics. 14(2):1-9.

28. RODRÍGUEZ, J.; PRIETO, S.; ORTIZ, L.; WIESNER, C.; DÍAZ, M.; CORREA, C. 2006. Descripción matemática con dimensiones fractales de células normales y con anormalidades citológicas de cuello uterino. Rev. Cienc. Salud. 4(2):58-63.

29. RODRÍGUEZ, J.; PRIETO, S.; CORREA, C.; POSSO, H.; BERNAL, P.; PUERTA, G.; VITERY, S.; ROJAS, I. 2010. Generalización fractal de células preneoplásicas y cancerígenas del epitelio escamoso cervical. Una nueva metodología de aplicación clínica. Rev. Fac. Med. 18(2):173-181.

30. RODRÍGUEZ, J. 2011. Nuevo método fractal de ayuda diagnóstica para células preneoplásicas del epitelio escamoso cervical. Rev. U.D.C.A Act. \& Div. Cient. 14(1):15-22.

31. RODRÍGUEZ, J.; PRIETO, S.; TABARES, L.; RUBIANO, A.; PRIETO, I.; DOMÍNGUEZ, D.; PATIÑO, O.; MEJÍA, M.; RAMÍREZ, L. 2013. Evolución de células de cuello uterino desde normales hasta atipias escamosas de significado indeterminado (ASCUS) con geometría fractal. Rev. U.D.C.A Act. \& Div. Cient. 16(2): 303-311.

32. RODRÍGUEZ, J.; PRIETO, S.; MELO, M.; DOMÍNGUEZ, D.; CARDONA, D.M.; CORREA, C.; LÓPEZ, F.; RODRÍGUEZ, L. 2014a. Simulación de rutas de alteración de células de cuello uterino desde el estado normal hasta lesion intraepitelial de bajo grado. Rev. U.D.C.A Act. \& Div. Cient. 17(1):5-12.

33. RODRÍGUEZ, J.; PRIETO, S.; CORREA, C.; SORACIPA, Y.; POLO, F.; PINILLA, L.; BLANCO, V.; RODRÍGUEZ, A. 2014b. Metodología diagnóstica geométrica fractal y euclidiana de células de cuello uterino. IATREIA. 27(1):5-13.

34. SANKAR, D.; THOMAS, T. 2010. A new fast Fractal modeling approach for the detection of microcalcifications in mammograms. J. Digit. Imaging. 23(5):538546.

35. SCHMIDT, J.L.; HENRIKSEN, J.C.; MCKEON, D.M.; SAVIK, K.; GULBAHCE, H.E.; PAMBUCCIAN, S.E. 2008. Visual estimates of nucleus-to-nucleus ratios: 
can we trust our eyes to use the Bethesda ASCUS and LSIL size criteria? Cancer. 114(5):287-293.

36. SOKOLOV, I.; DOKUKIN, M.E. 2017. Fractal Analysis of Cancer Cell Surface. En: Zeineldin, R. (ed). Cancer Nanotechnology: Methods and Protocols. Ed. Springer New York (New York). p.229-245.

37. STĘPIEŃ, R.; STĘPIEŃ, P. 2010. Analysis of contours of tumor masses in mammograms by Higuchi's fractal dimension. Biocybern. Biomed. Eng. 30(4):49-56.
38. VELÁSQUEZ, J.; PRIETO, S.; CATALINA, C.; DOMINGUEZ, D.; CARDONA, D.M.; MELO, M. 2015. Geometrical nuclear diagnosis and total paths of cervical cell evolution from normality to cancer. J. Cancer Res. Therapeutics. 11(1):98-104.

Recibido: Febrero 28 de 2016

Aceptado: Marzo 21 de 2017

Cómo citar:

Rodríguez, J.; Sánchez, M.; Barrios, F.; Soracipa, Y. 2017. Geometría fractal y euclidiana aplicada al diagnóstico de grados de lesión de células de cuello uterino. Rev. U.D.C.A Act. \& Div. Cient. 20(1): 13-21. 